\title{
Aminoglycosides for the treatment of septic shock: a propensity-based study
}

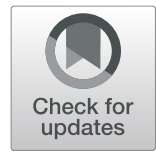

Jean-François Llitjos ${ }^{1,2,3^{*}}$, Simon Meslin ${ }^{1}$, Swann Bredin', Matthieu Jamme ${ }^{4,5}$ and Frédéric Pène ${ }^{1,2,3}$

Keywords: Septic shock, Aminoglycosides, Antibiotics, Intensive care unit, Bacterial resistance, Bacteria

The adequacy of initial antimicrobial treatment is a strong determinant of prognosis in septic shock. The prototypic synergistic combination of beta-lactams with aminoglycosides appears as an attractive therapeutic option, but its actual benefit remains elusive $[1,2]$. We took advantage of a large comprehensive cohort of septic shock to address the impact of aminoglycosides on mortality, with respect to their pharmacodynamic and pharmacokinetic properties.

We performed a retrospective single-center study over a 9-year period (2008-2016) of patients admitted to the intensive care unit (ICU) for septic shock, defined as microbiologically proven or clinically suspected infection associated with acute circulatory failure requiring vasopressors. The primary endpoint was in-ICU mortality. Patients treated or non-treated with aminoglycosides were matched in a 1:1 ratio using a logistic regressionbased propensity score including the following variables: age, gender, comorbid conditions, SAPS2, source of infection, biological findings, and organ supports at admission. Accuracy of aminoglycoside administration was characterized by the loading dose (recommended as $30 \mathrm{mg} / \mathrm{kg}$ amikacin or $6 \mathrm{mg} / \mathrm{kg}$ gentamycin/tobramycin) and the peak serum concentration $\left(C_{\text {peak }}\right)$ (targets recommended as $\geq 60 \mathrm{mg} / \mathrm{L}$ amikacin or $\geq 30 \mathrm{mg} / \mathrm{L}$

\footnotetext{
* Correspondence: jllitjos@gmail.com

This work was performed in: Service de médecine intensive-réanimation, Hôpital Cochin, Hôpitaux Universitaires Paris-Centre, Assistance Publique Hôpitaux de Paris, Paris, France

'Service de médecine intensive-réanimation, Hôpital Cochin, Hôpitaux Universitaires Paris-Centre, Assistance Publique - Hôpitaux de Paris, 27 rue du Faubourg Saint-Jacques, 75014 Paris, France

2Université de Paris, Paris, France

Full list of author information is available at the end of the article
}

gentamicin/tobramycin). Determinants of mortality were investigated in cause-specific proportional hazard model.

Among the 1040 patients, 616 (59\%) were administered a primary antibiotic combination regimen of beta-lactam with amikacin (379 patients, 62\%), gentamycin (229 patients, 37\%), or tobramycin (8 patients, $1 \%$ ). The overall mortality rate was $35 \%$. The propensity score-based matching process resulted in two cohorts of 348 patients with and without aminoglycosides (Table 1). Using the SAPS- 2 score, the severity was comparable between the two groups after matching (68 points $(52-85)$ in the aminoglycoside group versus 65 points (51-80) in the nonaminoglycoside group $(p=0.17))$. Among patients with microbiologically documented infections, the adequacy of the initial antibiotic regimen increased from $82 \%$ with single beta-lactam to $92 \%$ with combination regimen ( $p=$ 0.01 ). In combination-treated patients, $74 \%$ of documented pathogens were susceptible to both antibiotics whereas $12 \%$ were only susceptible to aminoglycosides. Loading doses of the first aminoglycoside infusion were appropriate in $21 \%$ of amikacin-treated and $27 \%$ of gentamycin/tobramycin-treated patients. Hence, only $18 \%$ of patients with available $\mathrm{C}_{\text {peak }}$ measurements achieved recommended concentration targets (30\% for amikacin while none for gentamycin/tobramycin) (Fig. 1). Furthermore, it is important to take into account that pneumonia is the main source of septic shock treated with aminoglycosides whereas their diffusion is poor in lung tissue.

Aminoglycoside treatment was associated with worse outcomes, including increased requirements for renal replacement therapy during the ICU stay and higher creatinine levels at the time of ICU discharge, and trend towards increased in-ICU mortality (Table 1). Mortality rates 
Table 1 Characteristics of septic shock patients treated or not with aminoglycosides before and after matching on the propensity score

\begin{tabular}{|c|c|c|c|c|c|c|}
\hline \multirow[t]{2}{*}{ Variables } & \multicolumn{2}{|l|}{ Before matching } & \multirow[t]{2}{*}{$p$} & \multicolumn{2}{|l|}{ After matching } & \multirow[t]{2}{*}{$p$} \\
\hline & $\begin{array}{l}\text { Aminoglycosides } \\
(n=616)\end{array}$ & $\begin{array}{l}\text { No aminoglycosides } \\
(n=424)\end{array}$ & & $\begin{array}{l}\text { Aminoglycosides } \\
(n=348)\end{array}$ & $\begin{array}{l}\text { No aminoglycosides } \\
(\mathrm{n}=348)\end{array}$ & \\
\hline Age, years & $70.2(58.2-79.7)$ & $67.5(56.5-77.5)$ & 0.02 & $70.2(56.6-79)$ & $66.9(55.9-76.9)$ & 0.06 \\
\hline Male gender & $386(62)$ & $278(65)$ & 0.36 & $227(65)$ & $228(65)$ & 1 \\
\hline Immunosuppression & $249(40)$ & $135(31)$ & 0.005 & $127(37)$ & $105(30)$ & 0.09 \\
\hline Neutropenia & $91(15)$ & $38(9)$ & 0.006 & $41(12)$ & $30(9)$ & 0.21 \\
\hline \multicolumn{7}{|l|}{ Characteristics on ICU admission } \\
\hline SAPS2, points & $70(53-87)$ & $65(51-80)$ & 0.002 & $68(52-85)$ & $65(51-80)$ & 0.17 \\
\hline Source of infection & & & & & & 0.29 \\
\hline Lung & $241(39)$ & $248(58)$ & $<0.001$ & $169(48)$ & $202(58)$ & \\
\hline Digestive & $82(13)$ & $46(11)$ & & $44(13)$ & $37(11)$ & \\
\hline Urinary & $101(17)$ & $24(5)$ & & $24(7)$ & $17(5)$ & \\
\hline Skin and soft tissue & $57(9)$ & $24(5)$ & & $30(9)$ & $23(7)$ & \\
\hline Catheter & $33(5)$ & $12(3)$ & & $13(4)$ & $9(3)$ & \\
\hline Others & $29(5)$ & $23(5)$ & & $20(6)$ & $21(6)$ & \\
\hline Unknown & $73(12)$ & $47(11)$ & & $48(14)$ & $39(12)$ & \\
\hline $\begin{array}{l}\text { Microbiological } \\
\text { documentation }\end{array}$ & $419(68)$ & $263(62)$ & 0.05 & $226(65)$ & $228(66)$ & 0.93 \\
\hline Bacteremia & $241(39)$ & $106(25)$ & $<0.001$ & $114(33)$ & $90(26)$ & 0.055 \\
\hline \multicolumn{7}{|l|}{ Microorganisms } \\
\hline Gram-negative bacteria & $290(47)$ & $131(31)$ & $<0.001$ & $137(60)$ & $102(45)$ & 0.003 \\
\hline Gram-positive bacteria & $120(19)$ & $120(28)$ & & $85(38)$ & $114(50)$ & \\
\hline Fungi & $9(1)$ & $10(2)$ & & $4(2)$ & $11(4.5)$ & \\
\hline Mycobacteria & $0(0)$ & $2(0.5)$ & & $0(0)$ & $1(0.5)$ & \\
\hline \multicolumn{7}{|l|}{ Biological findings } \\
\hline Serum protein level, g/L & $56(49-64)$ & $60(53-68)$ & $<0.001$ & $58(51-66)$ & $60.5(53-68)$ & 0.009 \\
\hline $\begin{array}{l}\text { Serum creatinine level, } \\
\mu \mathrm{mol} / \mathrm{L}\end{array}$ & $144(95-228)$ & $131(80-201)$ & 0.007 & $139(84-225)$ & $132(82-206)$ & 0.23 \\
\hline \multicolumn{7}{|l|}{ ICU management at day 1} \\
\hline First 24-h fluid balance, $\mathrm{mL}$ & $2485(1000-4378)$ & $2088(717-3552)$ & $<0.001$ & $2358(900-4200)$ & $2136(900-3700)$ & 0.18 \\
\hline $\begin{array}{l}\text { Renal replacement therapy } \\
\text { at day } 1\end{array}$ & $110(18)$ & 59 (14) & 0.13 & $58(17)$ & $52(15)$ & 0.60 \\
\hline $\begin{array}{l}\text { Norepinephrine amount at } \\
\text { day } 1, \mathrm{mg}\end{array}$ & $28.2(8.5-73)$ & $18.2(5.3-48.2)$ & $<0.001$ & $25(5.8-55.2)$ & $20(6.9-50.3)$ & 0.20 \\
\hline \multicolumn{7}{|l|}{ Aminoglycosides treatment } \\
\hline $\begin{array}{l}\text { Administration prior to ICU } \\
\text { admission }\end{array}$ & $116(19)$ & & & $50(14)$ & & \\
\hline Amikacin & $379(62)$ & & & $218(63)$ & & \\
\hline Loading dose, mg/kg & $19.7(17.2-23.6)$ & & & $20(17-24)$ & & \\
\hline $\begin{array}{l}\text { Recommended loading } \\
\text { dose }\end{array}$ & $78(21)$ & & & $51(23)$ & & \\
\hline Median $C_{\text {peak }}, \mathrm{mg} / \mathrm{L}$ * & $52.4(34.8-61)$ & & & $47.2(35.7-60.6)$ & & \\
\hline Recommended target & $36(33)$ & & & $18(30)$ & & \\
\hline Gentamicin/Tobramycin & $237(38)$ & & & $130(37)$ & & \\
\hline Loading dose, mg/kg & $4.7(4.2-6.1)$ & & & $5.4(4.2-6)$ & & \\
\hline Recommended loading & $63(27)$ & & & $40(30)$ & & \\
\hline
\end{tabular}


Table 1 Characteristics of septic shock patients treated or not with aminoglycosides before and after matching on the propensity score (Continued)

\begin{tabular}{|c|c|c|c|c|c|c|}
\hline \multirow[t]{2}{*}{ Variables } & \multicolumn{2}{|l|}{ Before matching } & \multirow[t]{2}{*}{$p$} & \multicolumn{2}{|l|}{ After matching } & \multirow[t]{2}{*}{$p$} \\
\hline & $\begin{array}{l}\text { Aminoglycosides } \\
(n=616)\end{array}$ & $\begin{array}{l}\text { No aminoglycosides } \\
(n=424)\end{array}$ & & $\begin{array}{l}\text { Aminoglycosides } \\
(n=348)\end{array}$ & $\begin{array}{l}\text { No aminoglycosides } \\
(\mathrm{n}=348)\end{array}$ & \\
\hline \multicolumn{7}{|l|}{ dose } \\
\hline Median $C_{\text {peak }}, \mathrm{mg} / \mathrm{L}^{*}$ & $15.2(10.6-19.9)$ & & & $14.7(10.4-19.6)$ & & \\
\hline Recommended target & $4(6)$ & & & $0(0)$ & & \\
\hline \multicolumn{7}{|l|}{ Overall ICU management } \\
\hline $\begin{array}{l}\text { Invasive mechanical } \\
\text { ventilation }\end{array}$ & $500(81)$ & $366(86)$ & 0.03 & $292(84)$ & $305(88)$ & 0.19 \\
\hline Renal replacement therapy & $319(52)$ & $162(38)$ & $<0.001$ & $179(51)$ & $131(38)$ & $<0.001$ \\
\hline \multicolumn{7}{|l|}{ Outcomes } \\
\hline $\begin{array}{l}\text { Creatininemia at day } 3 \text {, } \\
\mu \mathrm{mol} / \mathrm{L}\end{array}$ & $85(50-153)$ & $85(51-152.2)$ & 0.71 & $91(54-158.5)$ & $89.5(54.75-158.2)$ & 0.86 \\
\hline $\begin{array}{l}\text { Creatininemia at discharge, } \\
\mu \mathrm{mol} / \mathrm{L}\end{array}$ & $90(58-173)$ & $83(54.75-144.2)$ & 0.019 & $92.5(58-177)$ & $79.5(54-137.5)$ & 0.024 \\
\hline End-of-life decision & $136(22)$ & $117(28)$ & 0.047 & $52(15)$ & $69(20)$ & 0.11 \\
\hline 7-day mortality & $142(23)$ & $75(18)$ & 0.03 & $65(19)$ & $51(15)$ & 0.12 \\
\hline In-ICU mortality & $229(37)$ & $140(33)$ & 0.19 & $126(36)$ & $103(29)$ & 0.076 \\
\hline
\end{tabular}

*Among patients with available $C_{\text {peak }}$ before matching: amikacin: 108 (28\%) patients and gentamycin/tobramycin: 65 (27\%) patients and after matching: amikacin: $60(27 \%)$ patients and gentamycin/tobramycin: 38 (29\%) patients

of aminoglycoside-treated and aminoglycoside-untreated patients with microbiologically documented infections were not different (34\% and $31 \%$, respectively). In aminoglycoside patients who achieved the target concentration peak, the mortality was $28 \%$ whereas it was $33 \%$ in patients who did not $(p=0.76)$. After multivariate adjustment, aminoglycoside treatment was no longer associated with mortality (CSH 1.1; 95\%CI 0.90-1.55, $p=0.25$ ). Furthermore, aminoglycoside treatment did not impact on mortality in the relevant subgroups of neutropenic or bacteremic patients (CSH 1.11; 95\%CI 0.75-1.62, $p=0.61$ and $\mathrm{CSH} 1.03$; 95\%CI 0.64-1.66, $p=0.91$, respectively).

Aminoglycosides harbor potent antimicrobial properties including bactericidal activity, synergy with betalactams, post-antibiotic effect, and broadening the antibacterial spectrum [3]. However, the evidence of benefit in septic shock is scarce, based on controversial meta-analysis and retrospective studies $[1,2,4]$. Despite
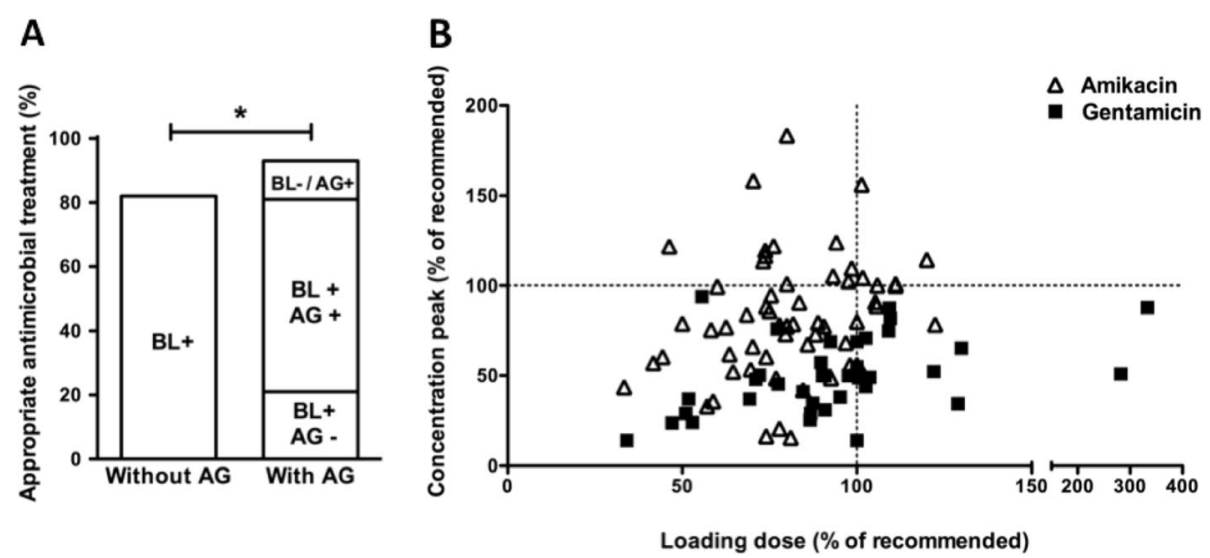

Fig. 1 Pharmacodynamic and pharmacokinetic parameters associated with aminoglycoside treatment. a Adequacy of antimicrobial treatment among propensity score-matched patients with microbiologically documented infections treated or not with aminoglycosides BL+: beta-lactam efficient, BL-: beta-lactam non efficient, AG-: aminoglycosides non efficient and AG+: aminoglycosides efficient. ${ }^{*} p=0.01$. $\mathbf{b}$ Relation between loading doses of aminoglycosides and concentration peak $\left(C_{\text {peak }}\right)$ among propensity score-matched patients. Loading doses and $C_{\text {peak }}$ are expressed in percentage of recommended values. The horizontal line represents the value of the recommended $C_{\text {peak }}$ for each aminoglycoside, and the vertical line depicts the value of the recommended loading dose 
the combination antibiotherapy improved the adequacy of initial antibiotic treatment, it did not translate into improved survival. However, the high incidence of aminoglycosides underdosing argues for accurate antimicrobial drug monitoring in further interventional trials [5].

\section{Acknowledgements}

None

\section{Authors' contributions}

JFL and FP designed the study. MJ performed the statistics. JFL, SM, and SB collected the date. All the authors wrote the manuscript. The authors read and approved the final manuscript.

\section{Funding}

None

\section{Availability of data and materials}

Yes

\section{Ethics approval and consent to participate}

The ethics committee of the French Intensive Care Society approved the study and waived the need for patients' consents due to its retrospective observational design (ref. CE SRLF, \#16-30).

\section{Consent for publication}

Not applicable

\section{Competing interests}

None to declare

\section{Author details}

'Service de médecine intensive-réanimation, Hôpital Cochin, Hôpitaux Universitaires Paris-Centre, Assistance Publique - Hôpitaux de Paris, 27 rue du Faubourg Saint-Jacques, 75014 Paris, France. ${ }^{2}$ Université de Paris, Paris, France. ${ }^{3}$ Institut Cochin, INSERM U1016, CNRS UMR 8104, Paris, France. ${ }^{4}$ Réanimation médico-chirurgicale, hôpital Poissy Saint-Germain, Poissy, France. ${ }^{5}$ INSERM U-1018, CESP, Team 5 (EpReC, Renal and Cardiovascular Epidemiology), Université Versailles Saint-Quentin, Villejuif, France.

Received: 2 June 2020 Accepted: 14 September 2020

Published online: 21 September 2020

\section{References}

1. Paul M, Lador A, Grozinsky-Glasberg S, Leibovici L. Beta lactam antibiotic monotherapy versus beta lactam-aminoglycoside antibiotic combination therapy for sepsis. Cochrane Database Syst Rev 2014;(1):CD003344..

2. Kumar A, Safdar N, Kethireddy S, Chateau D. A survival benefit of combination antibiotic therapy for serious infections associated with sepsis and septic shock is contingent only on the risk of death: a meta-analytic/ meta-regression study. Crit Care Med. 2010;38(8):1651-64.

3. Martinez JA, Cobos-Trigueros N, Soriano A, Almela M, Ortega M, Marco F, et al. Influence of empiric therapy with a -lactam alone or combined with an aminoglycoside on prognosis of bacteremia due to gram-negative microorganisms. Antimicrob Agents Chemother. 2010;54(9):3590-6.

4. Legrand M, Max A, Peigne V, Mariotte E, Canet E, Debrumetz A, et al. Survival in neutropenic patients with severe sepsis or septic shock. Crit Care Med. 2012;40(1):43-9.

5. de Montmollin E, Bouadma L, Gault N, Mourvillier B, Mariotte E, Chemam S, et al. Predictors of insufficient amikacin peak concentration in critically ill patients receiving a $25 \mathrm{mg} / \mathrm{kg}$ total body weight regimen. Intensive Care Med. 2014;40(7):998-1005.

\section{Publisher's Note}

Springer Nature remains neutral with regard to jurisdictional claims in published maps and institutional affiliations.

Ready to submit your research? Choose BMC and benefit from:

- fast, convenient online submission

- thorough peer review by experienced researchers in your field

- rapid publication on acceptance

- support for research data, including large and complex data types

- gold Open Access which fosters wider collaboration and increased citations

- maximum visibility for your research: over $100 \mathrm{M}$ website views per year

At BMC, research is always in progress.

Learn more biomedcentral.com/submissions 